\title{
Effect of contact geometry on the friction of acrylamide hydrogels with different surface structures
}

\author{
Wenrui LIU ${ }^{1,2}$, Rok SIMIČ ${ }^{2, *}$, Yuhong LIU ${ }^{1, *}$, Nicholas D. SPENCER ${ }^{2}$ \\ ${ }^{I}$ State Key Laboratory of Tribology, Tsinghua University, Beijing 100084, China \\ ${ }^{2}$ Laboratory for Surface Science and Technology, Department of Materials, ETH Zurich, 8093 Zurich, Switzerland \\ Received: 27 July 2020 / Revised: 14 September 2020 / Accepted: 25 September 2020 \\ (C) The author(s) 2020 .
}

\begin{abstract}
Polyacrylamide (PAAm) hydrogels with brush-covered or crosslinked surfaces were produced and their tribological behavior was studied over a wide range of sliding speeds for two different contact geometries: sphere-on-flat and flat-pin-on-flat. Irrespective of the contact geometry, the brushy hydrogel surfaces displayed up to an order of magnitude lower coefficients of friction $\mu$ (COF) compared to the crosslinked surfaces, even achieving superlubricity $(\mu<0.01)$. In general, a hydrogel sphere showed a lower coefficient of friction than a flat hydrogel pin at a similar contact pressure over the entire range of sliding speeds. However, after normalizing the friction force by the contact area, the shear stress of hydrogels with either crosslinked or brushy surfaces was found to be similar for both contact geometries at low speeds, indicating that hydrogel friction is unaffected by the contact geometry at these speeds. At high sliding speeds, the shear stress was found to be lower for a sphere-on-flat configuration compared to a flat-pin-on-flat configuration. This can be attributed to the larger equivalent hydrodynamic thickness due to the convergent inlet zone ahead of the sphere-on-flat contact, which presumably enhances the water supply in the contact, promotes rehydration, and thus reduces the friction at high sliding speeds compared to that measured for the flat-pin-on-flat contact.
\end{abstract}

Keywords: hydrogels; contact geometry; aqueous lubrication; friction coefficient (COF); superlubricity

\section{Introduction}

Hydrogels are soft materials consisting of a threedimensional, cross-linked polymer network containing a large amount of water. The high water content (> $90 \mathrm{wt} \%$ ) in combination with good lubricating properties of hydrogels makes them good materials to construct polymeric analogues of articular cartilage [1-3] or other tissues subjected to rubbing, including the trachea [4], skin [5, 6], and blood vessels [7]. In order to develop hydrogels that would closely mimic natural lubrication systems and be used for medical applications, it is of great importance to understand their lubrication mechanisms. Numerous studies have been conducted to investigate the tribological properties of hydrogels. However, the dissipation mechanisms of hydrogel friction are not yet completely understood, which impedes the achievement of any improvement in their lubrication performance. Currently, hydrogel friction is found to largely depend on the normal load, the relative sliding speed, and the surface structure of hydrogels.

Gong and coworkers have conducted a series of studies on the tribological properties of hydrogels [8-15]. Through comparative frictional tests of various hydrogels, they found that hydrogel friction does not always follow Amonton's law [10, 11], according to which the frictional force is proportional to the

* Corresponding authors: Rok SIMIČ, E-mail: rok.simic@mat.ethz.ch; Yuhong LIU, liuyuhong@tsinghua.edu.cn 
normal load. As the normal load increased, the friction force of polyvinyl alcohol (PVA) hydrogels, for example, significantly increased, while that of Gellan hydrogels (a polysaccharide produced by the bacterium Sphingomonas elodea) was nearly independent of the normal load. The relationship between the hydrogel friction force and the normal load was found to follow a power law, $F_{\mathrm{f}} \propto W^{\partial}$, where $F_{\mathrm{f}}$ is the friction force, $W$ is the applied normal load and $\partial$ is the scaling exponent with a value between 0 and 1 $[1,16]$. The group of Sawyer has shown for polyacrylamide (PAAm) hydrogels that shear stress remains constant during sliding at different normal stresses, and that friction changes only upon load-induced changes in contact area [17].

The relative speed between sliding surfaces has been demonstrated to have a significant influence on hydrogel friction. Gong and coworkers observed that hydrogel friction had different speed-dependent trends at low and at high speeds, and proposed the adsorption-repulsion model [10-14], which depends on the type of interactions between the hydrogels and the sliding counterpart. In the model, the viscous force of the shearing layer always plays a dominant role in hydrogel friction when the interface interaction is repulsive during the sliding process. When the interface interaction is attractive, the hydrogel friction is attributed to two factors, which are the elastic deformation of adsorbed polymer chains and the viscous force of the shearing layer. In this case, the elastic deformation of polymer chains dominates friction at low speeds, while viscous force plays a leading role at high sliding speeds. The group of Sawyer investigated the effect of sliding speed using self-mated ("gemini") hydrogels and determined a transition speed in friction [18, 19]. They observed that at low sliding speeds hydrogels displayed a low, speed-independent coefficient of friction (COF), while at high speeds the COF increased with increasing sliding speed. The relationship obeyed a power law, $\mu \propto v^{0.5}$, where $\mu$ is the COF and $v$ is the relative sliding speed. The transition speed of the friction behavior was considered to be closely related to the mesh size and relaxation time of the polymer network [20,21].

Hydrogel friction has also been shown to be highly dependent on the surface structure of the gels [10, 14, 15, 22-24]. It was found that a friction increase with increasing sliding speed is commonly observed with hydrogels with a crosslinked surface. A mechanism based on the hydrodynamic shearing of a nanoscopic lubricating film between the sliding surfaces was proposed to explain their tribological behavior [22]. However, hydrogels with a brushy surface were shown to have much lower friction over the same range of sliding speeds. Due to the sparse, brushy surface structure, which traps large amounts of water at the interface, the chances for direct polymer chain contact are lower and the hydrodynamic shearing thickness is presumably larger, reducing the friction. In these brushy, water-rich hydrogel surfaces, however, the friction was demonstrated to depend on water exudation and rehydration in the near-surface region [23]. When a brushy surface is exposed to sufficient constant normal load, it exudes water, increasing the polymer concentration near its surface, and causing it to behave similarly to a hydrogel with a crosslinked surface. Therefore, in typical friction experiments, in which a smaller top hydrogel counterpart is constantly in contact, its surface structure does not play a significant role in friction [23]. The friction seems to be predominantly determined by the surface structure of the larger hydrogel counterpart, where the migrating contact area allows for the surfaces to rehydrate during the out-of-contact period.

Contact geometry has been found to be a significant factor in friction $[25,26]$. Although numerous studies have been conducted to gain an understanding of hydrogel friction employing different materials, normal loads, and sliding speeds, very little is known about the effects of the contact geometry, which could play an important role during the sliding process. Most of the available literature has presented results of either flat-on-flat or sphere-on-flat contact. Although the non-conformal, sphere-on-flat contact is very commonly used in tribological studies of engineering materials, it can rarely be found in nature, where compliant contact pairs have relatively large contact areas, in order to facilitate low contact pressures. At the same time, the results obtained with such different contact geometries are difficult to compare, if the effects of different contact boundaries (trailing 
edge of a flat-pin-on-flat compared to a convergent inlet of a sphere-on-flat) are unknown. We have already shown that the continuous contact of a ring-on-flat displays similar friction as a flat-pin-on-flat hydrogel contact at sliding speeds between 0.1-20 $\mathrm{mm} / \mathrm{s}$ [23]. However, sphere-on-flat contact might facilitate water supply to the contact due to the convergent contact inlet when compared to a flat-on-flat contact geometry [27]. It can also be inferred that this effect would be more pronounced at higher sliding speeds, which would allow a thicker lubricating film and thus reduce the hydrodynamic friction.

Different contact geometries also differently affect the increase in contact pressure when the normal load is increased. For example, for a flat-pin-onflat configuration, the contact area is independent of the load, making the contact pressure directly proportional to the load. On the other hand, for a sphere-on-flat geometry, the contact area and the contact pressure change in accordance with the Hertzian contact theory [17]. These differences have typically not been discussed in the available hydrogeltribology literature. However, they might have a significant influence on hydrogel friction.

This paper thus aims to resolve the effects of contact geometry on the friction of hydrogels for two configurations-Flat-pin-on-flat and sphere-on-flat. The main hypothesis is that a convergent inlet in a sphere-on-flat contact facilitates hydrodynamic friction at high speeds and thus helps in reducing friction. Furthermore, bearing in mind the known differences in frictional behavior between different hydrogel surface structures, we have also compared the frictional behavior of two distinctly different hydrogel surfacesA brushy and a crosslinked surface. Experiments were performed under different contact loads to study the effect of contact pressure and contact area on frictional behavior.

\section{Materials and methods}

\subsection{Materials}

Acrylamide (AAm, Sigma-Aldrich, > 99\%) and N, N'-methylenebisacrylamide (bis-AAm, Sigma-Aldrich, $\geq 99.5 \%$ ) were selected as the monomer and crosslinker, respectively. Lithium phenyl-2,4,6-trimethylben- zoylphosphinate (LAP), which was synthesized via the Michaelis-Arbuzov reaction and subsequent solvolysis in our laboratory as described in Refs. [28-30], was used as the ultraviolet (UV) light initiator.

\subsection{PAAm hydrogel preparation}

For synthesizing PAAm hydrogels, $9.6 \mathrm{wt} \%$ of AAm, $0.4 \mathrm{wt} \%$ of bis-AAm, and $0.01 \mathrm{wt} \%$ of LAP were first dissolved in Milli-Q water during ultrasonic stirring. To produce hydrogel surfaces with different structures, the solutions were gelled in different mold materials. Glass petri dishes were selected to prepare hydrogel discs with a dense, crosslinked surface, while polystyrene (PS) petri dishes were selected to prepare hydrogel discs with a sparse, brushy surface [22, 23]. By using spacers between two molding surfaces, we prepared hydrogel sheets of 2-mm and 5-mm thickness, which served to produce flat upper and lower tribological specimens, respectively. A roundbottomed glass reagent tube with a radius of curvature of around $9.5 \mathrm{~mm}$ was used to prepare hemispherical hydrogels with a dense, crosslinked surface that served as the upper specimen for the sphere-on-flat tribological tests. All molds were cleaned with acetone, ethanol, and Milli-Q water before the polymerization reaction. The molds with the solution were placed into a UV cross-linker (Stratalinker UV Cross-linker 2400, Stratagene Corp., La Jolla, CA, USA) and polymerization was carried out for $10 \mathrm{~min}$. The intensity of the UV light was about $1 \mathrm{~mW} \cdot \mathrm{cm}^{-2}$ and the wavelength was $365 \mathrm{~nm}$. After the polymerization, the synthesized PAAm hydrogels were removed from the molds and placed into a large amount of Milli-Q water for at least $48 \mathrm{~h}$ to remove unreacted monomers and to swell fully. Then, small discs of 10-mm diameter were punched out of the thin, glass-molded hydrogel slab to prepare the flat hydrogel pins. Thus, two contact geometries, flat-pin-on-flat, and sphere-onflat were used in the tests, as shown in Fig. 1. Since it was shown that the structure of the stationary contact does not affect the frictional properties [23], the top specimen, being either a flat pin or a sphere, always had a crosslinked surface. For the bottom specimen, hydrogel discs with a swollen thickness 


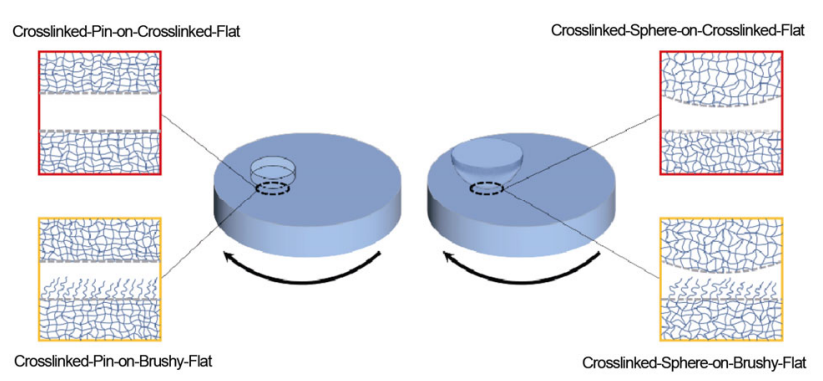

Fig. 1 Schematics of the hydrogel-hydrogel contact geometries used in the friction experiments. Two contact geometries, flatpin-on-flat, and sphere-on-flat were used with different sliding hydrogel surfaces.

of about $6 \mathrm{~mm}$, with a diameter around $50 \mathrm{~mm}$, and having either a crosslinked (glass-molded) or sparse, brushy (PS-molded) surface were used.

\subsection{Indentation tests}

Nanoindentation tests were performed using an atomic force microscope (AFM, MFP-3D, Asylum Research, Santa Barbara, USA) to identify the surface structures of differently molded PAAm surfaces. All experiments were performed with the samples fully immersed in water. The spring constant $k$ of the tipless, gold-coated cantilever (NSC-36, Mikromash, Bulgaria) was measured using the Sader method [31]. A 14- $\mu$ m-radius silica microsphere (GP0083, Whitehouse Scientic, Waverton, UK) was glued to the end of the tipless cantilever with a 2-component epoxy resin adhesive (UHU GmbH, Germany). The effective spring constant at the colloid position was calculated as $k^{\prime}=k\left(L / L^{\prime}\right)^{3}=1.28 \mathrm{~N} / \mathrm{m}$, where $L$ and $L^{\prime}$ are the distances from the base of the cantilever to the tip of the cantilever and the colloid position, respectively [32]. To calibrate the optical-lever sensitivity $S$, which is the ratio of cantilever deflection change $\Delta x$ and the photodiode signal change $\Delta U$, the probe was pressed against a silicon wafer in water. The indentation depth was calculated as $d=Z-x=Z-S U$, where $Z$ is the measured z-piezo displacement. The force was calculated as $F=k^{\prime} x$. The contact point was determined as the last data point lying within $2 \sigma$ from the zero-force line on the approach curve, where $\sigma$ is the root-mean-square value of the noise away from the surface $(\sim 20 \mathrm{pN})$. The approach speed was $1 \mu \mathrm{m} \cdot \mathrm{s}^{-1}$. All measurements were performed at room temperature $\left(25 \pm 2{ }^{\circ} \mathrm{C}\right)$. Force curves were obtained on different locations of each sample, to check for reproducibility. Results are presented as representative force-indentation curves. The first 1.5 $\mu \mathrm{m}$ of indentation curves were fitted using the Hertzian contact model [33]. For the hydrogel molded in PS molds, the fit was extrapolated over the entire indentation range, in order to show the softness of the outermost layer of the hydrogel, which then gradually stiffened with increasing indentation depth.

Macroindentation tests were performed using the Universal Mechanical Tester (UMT, Bruker, Massachusetts, USA) to measure the elastic moduli of the bulk hydrogels with a flat-punch and a spherical indenter. For this, an AISI 52100 steel cylinder with a diameter of $9.5 \mathrm{~mm}$ and an AISI 52100 steel ball with a radius of $9.5 \mathrm{~mm}$ were used. The elastic modulus of the bulk was measured for the thick, glass-and PS-molded hydrogels to ensure that the bulk properties were similar and the hydrogels only differed in their surface structure. The Hertzian model was used to describe sphericalindenter indentations up to a depth of $0.3 \mathrm{~mm}$, corresponding to $5 \%$ of the hydrogel slab thickness. To check the validity of the Hertzian fit, we have also applied the Winkler model [34] to the entire indentation curve, assuming a slab thickness of $6 \mathrm{~mm}$. Moreover, a linear fit was used to describe indentations with a cylindrical, flat-punch indenter. Due to the large diameter $(9.5 \mathrm{~mm})$ of the punch with respect to the hydrogel thickness $(H \approx 6 \mathrm{~mm})$, the modulus was determined assuming a uniform linear compression by using the following equation:

$$
W=A E \Delta x / H
$$

where $W$ is the normal force, $A$ is the contact area, $\Delta x$ is the change in thickness upon compression, $H$ is the nominal thickness of the hydrogel sample, and $E$ is the effective modulus of elasticity of the friction pairs. The $E$ is defined as

$$
E=1 /\left(\frac{1-\varepsilon_{1}^{2}}{E_{1}}+\frac{1-\varepsilon_{2}^{2}}{E_{2}}\right)
$$

where $\varepsilon_{i}$ denotes the Poisson's ratio for material $i$, and $E_{i}$ denotes the elasticity modulus of material $i$. 


\subsection{Tribological evaluations}

A parallel-plate rheometer (ARES-G2, TA Instruments, Delaware, USA) was used to perform unidirectional sliding experiments under a load of $0.5 \mathrm{~N}$. Either a flat hydrogel pin or a hydrogel sphere was glued to the top parallel plate of the rheometer using a cyanoacrylate-based superglue (Pattex, Henkel AG \& Co. KGaA, Düsseldorf, Germany), with their centers at a radius of $10 \mathrm{~mm}$ from the axis of rotation. The bottom hydrogel flat was glued to a PS petri dish and covered with ultrapure water. The dish was then fixed to the lower parallel plate of the rheometer using a thin layer of double-sided adhesive tape. Due to the thin hydrogel pin, the lateral expansion under a load of $0.5 \mathrm{~N}$ was assumed to be negligible and the contact area was considered to be equal to the nominal cross-sectional area of the pin. Thus, the corresponding contact pressure between the pin and the flat was around $6 \mathrm{kPa}$. The contact area between the hydrogel sphere and the flat were calculated according to the Hertzian contact model:

$$
D=2 \cdot \sqrt[3]{\frac{3 W R}{4 E}}
$$

where $D$ is the diameter of the contact area, $W$ is the load, $E$ is the elastic modulus, and $R$ is the radius of the hydrogel sphere. Considering the elastic modulus of $95 \mathrm{kPa}$ (as obtained from the indentation tests) and a Poisson's ratio of 0.5, the calculated diameter $D$ was around $8 \mathrm{~mm}$ and the corresponding average contact pressure was around $10 \mathrm{kPa}$ at a load of $0.5 \mathrm{~N}$. The tests with the rheometer were performed over a range of angular velocities from 0.005 to $0.5 \mathrm{rad} \cdot \mathrm{s}^{-1}$, corresponding to sliding speeds from 0.05 to $5 \mathrm{~mm} \cdot \mathrm{s}^{-1}$. Above these sliding speeds, however, the load control of the system was too slow for effective compensation of the small sample-thickness variation, resulting in significant variation of the load and consequent irreproducibility of the results. Therefore, the experiments with the rheometer were limited to a maximum velocity of $5 \mathrm{~mm} \cdot \mathrm{s}^{-1}$.

In order to extend the range of sliding speeds, the frictional tests were conducted using the UMT in a unidirectional configuration. The radius of rotation was set to $10 \mathrm{~mm}$ and the rotational speed was adjusted to cover a range of linear speeds from 0.5 to $200 \mathrm{~mm} \cdot \mathrm{s}^{-1}$. Combined with the tests using the rheometer, the friction experiments were performed at sliding speeds ranging over almost 4 orders of magnitude. For the measurements using the UMT tribometer, the normal force and the friction force were simultaneously measured every $0.001 \mathrm{~s}$, and by averaging every 1,000 points, a similar data frequency of $1 \mathrm{~Hz}$ was obtained as with the rheometer. The duration of each experiment, using either the rheometer or the UMT tribometer, was set to allow several full rotations of the sample, and the characteristic COF was determined from the last, equilibrated part of the friction curve. The temperature during all the friction experiments was approximately $25{ }^{\circ} \mathrm{C}$. The tests were performed at different locations of the hydrogel samples and in different orientations and at least three times under each set of conditions. The results are presented as an average value with error bars corresponding to one standard deviation. We observed no visible changes to either the pins or the hydrogel flats throughout the experiments. The friction remained constant over time, which indicated that either no damage or minimal damage to the hydrogel surface occurred under these sliding conditions.

\section{Results and discussion}

\subsection{Indentation tests}

The experimental nanoindentation data for the crosslinked (glass-molded) and brushy (PS-molded) hydrogel surface are shown in Fig. 2. The Hertzian contact model fits of the experimental data over the first $1.5 \mu \mathrm{m}$ are also shown. It can be seen that the force-indentation curve for the glass-molded hydrogel surface can be well described by the Hertzian contact model, which indicates that a homogeneously crosslinked structure with a constant elastic modulus was present at the hydrogel surface. However, a very different force-indentation curve was observed for the PS-molded hydrogel surface. Fitting the Hertzian contact model to the initial $1.5 \mu \mathrm{m}$ of indentation 


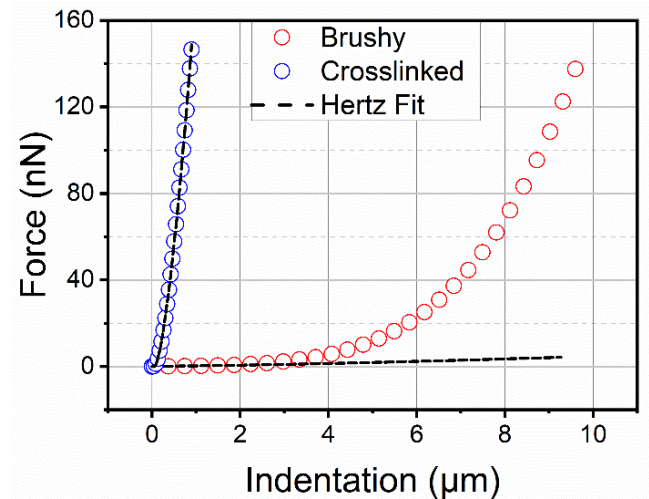

Fig. 2 Experimental indentation data generated by nanoindentation on glass-molded (crosslinked) and PS-molded (brushy) hydrogel surfaces and the corresponding fits of the initial $1.5 \mu \mathrm{m}$ of the indentation data using the Hertzian contact model.

showed an elastic modulus lower than $0.1 \mathrm{kPa}$, indicating an extremely soft and sparse, presumably brushy, surface structure. The force curve was also observed to increase with indentation depth beyond this Hertzian regime, indicating that the hydrogel structure gradually densified and stiffened with increasing depth.
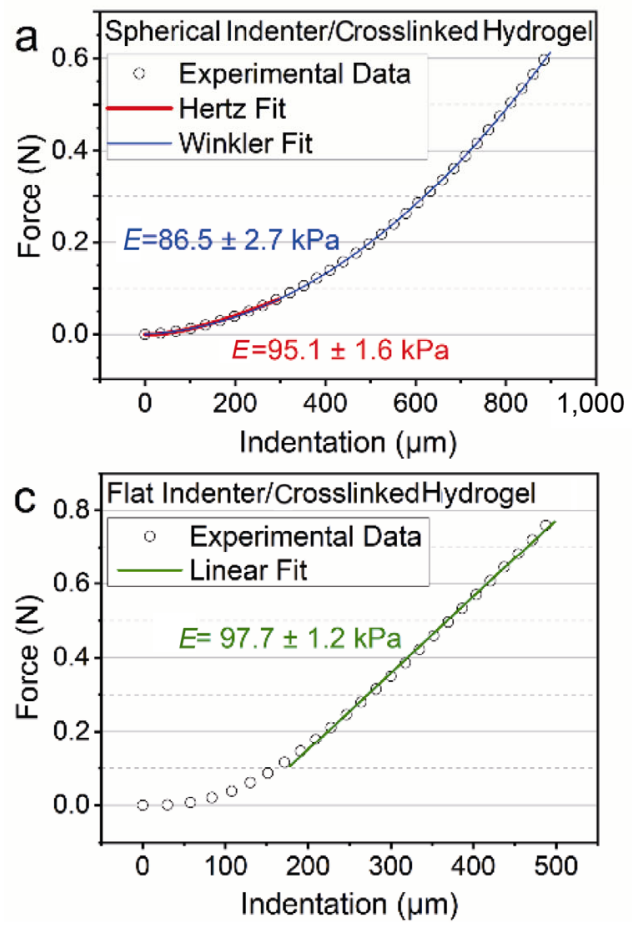

Assuming the molding surface only affected the hydrogel structure in the near-surface region, the differently molded hydrogels were expected to have the same elastic modulus within their bulk. In order to verify this, macro-indentation experiments with a spherical and a flat indenter were performed on both types of gels.

The experimental indentation data obtained by a spherical indenter are shown in Figs. 3(a) and 3(b). The Hertzian contact model assumes an indentation into an elastic half-space and is commonly applied to indentation depths that do not exceed $10 \%$ of the total thickness of the sample. Therefore, the Hertzian model was fitted to the initial $0.3 \mathrm{~mm}$ of the indentation on $6 \mathrm{~mm}$ thick samples. As shown in Figs. 3(a) and 3(b), it can be found that the initial force-indentation curves achieved on both glass-molded and PS-molded hydrogels could be well described by the Hertzian contact model. The elastic moduli of the glassmolded and PS-molded hydrogels were found to be $95.1 \pm 1.6 \mathrm{kPa}$ and $94.5 \pm 1.2 \mathrm{kPa}$, respectively,
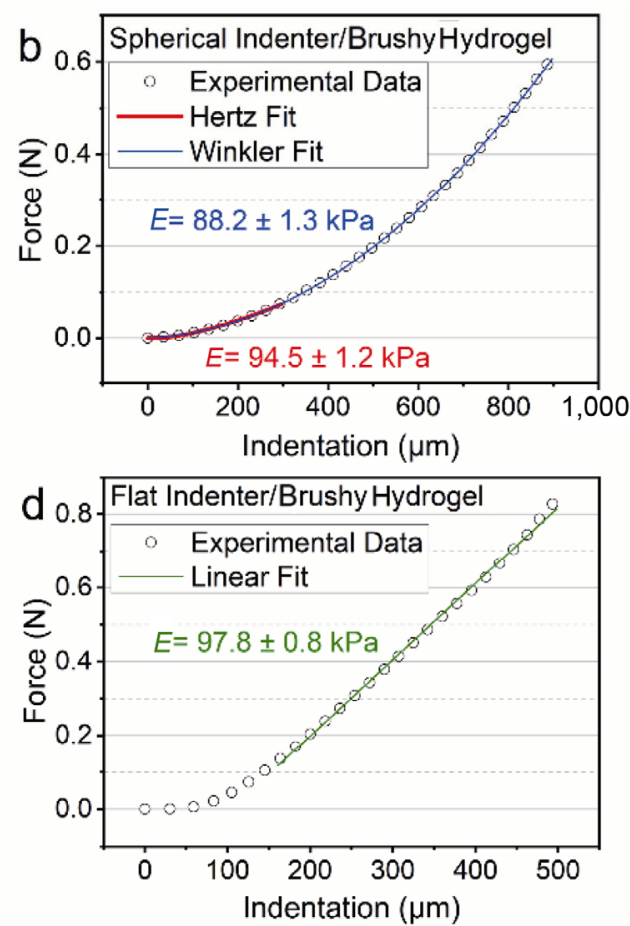

Fig. 3 Experimental macro-indentation data generated by a spherical indenter on (a) glass-molded (crosslinked) and (b) PS-molded (brushy) hydrogel surface, and a cylindrical, flat-punch indenter on (c) glass-molded (crosslinked) and (d) PS-molded (brushy) hydrogel surface. Corresponding fits of the data using the Hertzian and Winkler models for the spherical indenter and linear compression for the flat-punch indenter are also shown. 
indicating their similar bulk structure. Moreover, the similar moduli values also showed that the surface structures were limited to the initial few micrometers of depth and were thus undetectable during macroindentation measurements. The Winkler model, on the other hand, takes into account finite sample thickness and can be used to describe indentation processes, in which the thickness of the sample is comparable to the indenter radius. The forceindentation data could also be well described using the Winkler model over the entire indentation depth of about $0.9 \mathrm{~mm}$. This again is consistent with a homogeneous structure of the hydrogel bulk. Fitting the Winkler model, the elastic modulus of the glass-molded hydrogel had a value of $86.5 \pm$ $2.7 \mathrm{kPa}$ and that of the PS-molded hydrogel a value of $88.2 \pm 1.3 \mathrm{kPa}$, which were, as expected, very similar to the values obtained using the Hertzian model. On the other hand, the experimental indentation data obtained by a cylindrical, flat-punch indenter are shown in Figs. 3(c) and 3(d). After the initial transition to full contact with the flat indenter, a linear fit was used to describe the experimental data. The elastic modulus of the glass-molded hydrogel was found to be $97.7 \pm 1.2 \mathrm{kPa}$ and that of the PS-molded hydrogel had a value of $97.8 \pm 0.8 \mathrm{kPa}$. These results demonstrate that the PAAm hydrogels with different surface structures indeed had the same bulk structure.

\subsection{Tribological evaluations}

The COF as a function of speed for different contact geometries of PAAm hydrogels with different surfaces is shown in Fig. 4(a). It was found that the crosslinked hydrogels displayed the highest COF in a flat-pinon-flat configuration over the entire speed range. Hydrogel pins even suffered from breaking at sliding speeds above $20 \mathrm{~mm} \cdot \mathrm{s}^{-1}$, indicating that the shear stress at these sliding speeds exceeded the ultimate shear strength of the hydrogel. At sliding speeds below $1 \mathrm{~mm} \cdot \mathrm{s}^{-1}$, however, the COF was almost speedindependent with glass-molded hydrogels, with values between 0.1 and 0.2 . At sliding speeds above $1 \mathrm{~mm} \cdot \mathrm{s}^{-1}$, the COF showed a significant increase with increasing sliding speed, scaling as $\mu \propto v^{0.5}$, which is similar to the power value observed in other recent studies $[22,23,35,36]$. A similar trend was observed when sliding crosslinked hydrogels in a sphere-on-flat configuration. At sliding speeds below $5 \mathrm{~mm} \cdot \mathrm{s}^{-1}$, the COF showed only a moderate increase with increasing speed. At sliding speeds above $5 \mathrm{~mm} \cdot \mathrm{s}^{-1}$, however, the COF again scaled with sliding speeds with a power of around 0.5. This is similar to the speed-dependent behavior at high speeds with a sphere-on-flat contact observed in recent Refs. [18, 19, 37]. Due to the small, but significant difference between the contact areas of the flat-pin-on-flat and the sphere-on-flat configurations, we have normalized the friction force by the contact area and presented the shear stress as a function of sliding speed in Fig. 4(b). Similar shear-stress values were found for both contact geometries at sliding speeds below $5 \mathrm{~mm} \cdot \mathrm{s}^{-1}$. It was observed that at similar contact pressures the shear stress is the same for
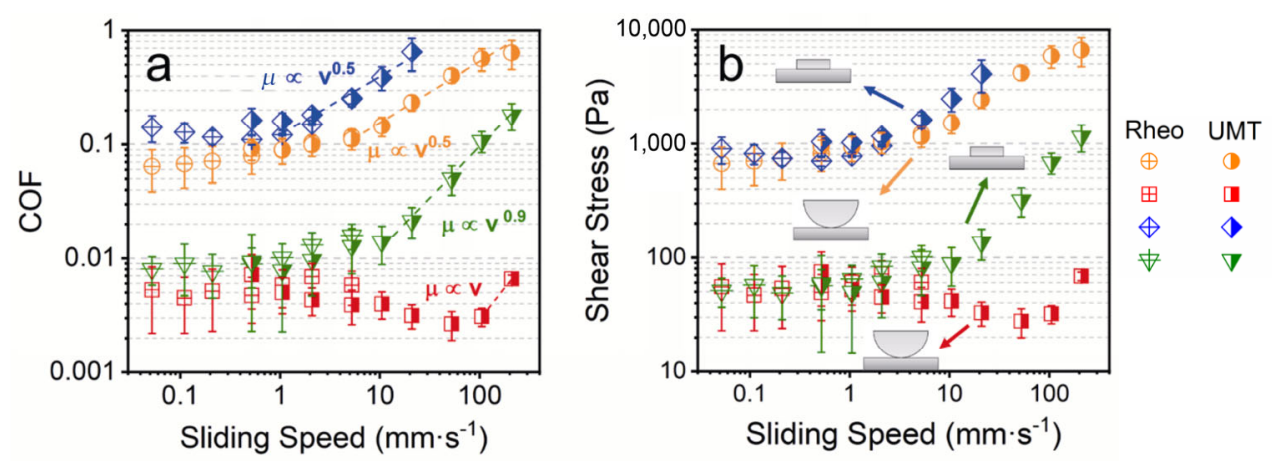

Sphere-on-Crosslinked-Flat

Sphere-on-Brushy-Flat

Pin-on-Crosslinked-Flat

Pin-on-Brushy-Flat

Fig. 4 (a) COF and (b) shear stress as a function of speed for different contact geometries of PAAm hydrogels with different surface structures. The tests performed with the rheometer are shown by empty symbols and the tests performed with the UMT are shown by semi-filled symbols. The contacts between a pin and a crosslinked surface are shown in blue, between a sphere and a crosslinked surface are shown in orange, between a pin and a brushy surface are shown in green, and between a sphere and a brushy surface are shown in red. 
both contact geometries, indicating that it was the contact area that dictates the friction force. At sliding speeds above $5 \mathrm{~mm} \cdot \mathrm{s}^{-1}$, the shear stress in the case of the flat-pin-on-flat geometry showed larger values compared to those of the sphere-on-flat geometry. The observed difference at high sliding speeds could originate from lubricating films of different thicknesses. In other words, the convergent inlet zone of the sphere-on-flat contact might enhance fluid entrainment and thus increase the shearingfilm thickness, which in turn would reduce the shear stress compared to that of the flat-pin-on-flat contact $[18,19]$.

In contrast to the hydrogels with the crosslinked surface, the COF values of the PAAm hydrogels with brushy surfaces were about an order of magnitude lower over the entire range of tested sliding speeds. As shown in Fig. 4(a), PS-molded hydrogels in a flat-pin-on-flat configuration also underwent a transition from low, speed-independent friction to a COF that was increasing with sliding speeds, similarly to the glass-molded hydrogels. At sliding speeds below $10 \mathrm{~mm} \cdot \mathrm{s}^{-1}$, the COF for the flat pin on the brushy flat was almost constant, with values between 0.008 and 0.015 . At sliding speeds above $10 \mathrm{~mm} \cdot \mathrm{s}^{-1}$, the hydrogel friction displayed a significant speed-dependent behavior, scaling as $\mu \propto v^{0.9}$. However, sliding a crosslinked spherical hydrogel surface over a brushy flat surface maintained superlubricity, with values between 0.002 and 0.007 over the entire range of sliding speeds. As shown in Fig. 4(a), it was found that the COF was almost constant at sliding speeds of up to 50-100 $\mathrm{mm} \cdot \mathrm{s}^{-1}$, where it started to increase almost proportionally with the sliding speed, i.e., scaling as $\mu \propto v$. Analyzing the shear stress over the full range of speeds, as shown in Fig. 4(b), it was found that the shear stress in the two contact geometries showed a similar value and remained almost constant with speed, below $5 \mathrm{~mm} \cdot \mathrm{s}^{-1}$. The similar shear stress at low speeds indicates, as for the crosslinked case, that hydrogel friction is unaffected by contact geometry at these speeds. At sliding speeds above $5 \mathrm{~mm} \cdot \mathrm{s}^{-1}$, the values of shear stress for flat and spherical contact showed significant differences. The shear stress of hydrogels with the brushy surface in a flat-pin-on-flat configuration increased rapidly with increasing sliding speed, while that in the sphereon-flat configuration seemed to have decreased at first and started increasing only at sliding speeds above $50-100 \mathrm{~mm} \cdot \mathrm{s}^{-1}$. This suggests that contact geometry also affects the high-speed friction of hydrogels with brushy surfaces.

Assuming the friction is governed by Newtonian shearing of water, the equivalent hydrodynamic thickness $h$, which includes the shearing layer and the depth of the flowing water within the sparse structure on the surface, was calculated by using Newton's law of viscous flow:

$$
h=\frac{A \eta U}{\mu W}
$$

where $\eta$ is dynamic viscosity and $U$ is the sliding speed. Considering the viscosity of the water to be $1 \mathrm{mPa} \cdot \mathrm{s}$ at $25{ }^{\circ} \mathrm{C}$, the effective hydrodynamic thickness was calculated over the whole range of sliding speeds and is displayed in Fig. 5. For hydrogels with crosslinked surfaces, the surface structure is too dense to allow significant water flow within the near- surface region, which is more similar to engineering surfaces. It can be seen that the theoretical equivalent hydrodynamic thickness in both contact geometries was more than $1 \mathrm{~nm}$ when the sliding speed exceeded $1 \mathrm{~mm} \cdot \mathrm{s}^{-1}$. Assuming that the surface roughness of the hydrogel resembles that of the mold, the $R_{q}$ value of the glass-molded hydrogels would be around $1 \mathrm{~nm}$ [23]. Calculating the $\lambda$ values as $\lambda=h / \sqrt{R_{q 1}^{2}+R_{q 2}^{2}}$, where $R_{q i}$ is the surface roughness of material $i$, reveals that a $\lambda$ value larger than 1 , which would indicate the presence of hydrodynamic effects, appears with sliding speeds higher than $1 \mathrm{~mm} \cdot \mathrm{s}^{-1}$. Notably, this speed was also the transition speed, above which the COF and shear stress of hydrogels with the crosslinked surface were observed to increase rapidly with increasing sliding speed. It can be inferred that the speed-dependent friction behavior at these high speeds may be closely related to hydrodynamic effects. Thus, as shown in Fig. 5, for the flat-pin-on-flat configuration between a crosslinked pin and a crosslinked surface, the hydrodynamic effect would be gradually enhanced with increasing sliding speed and become dominant at 


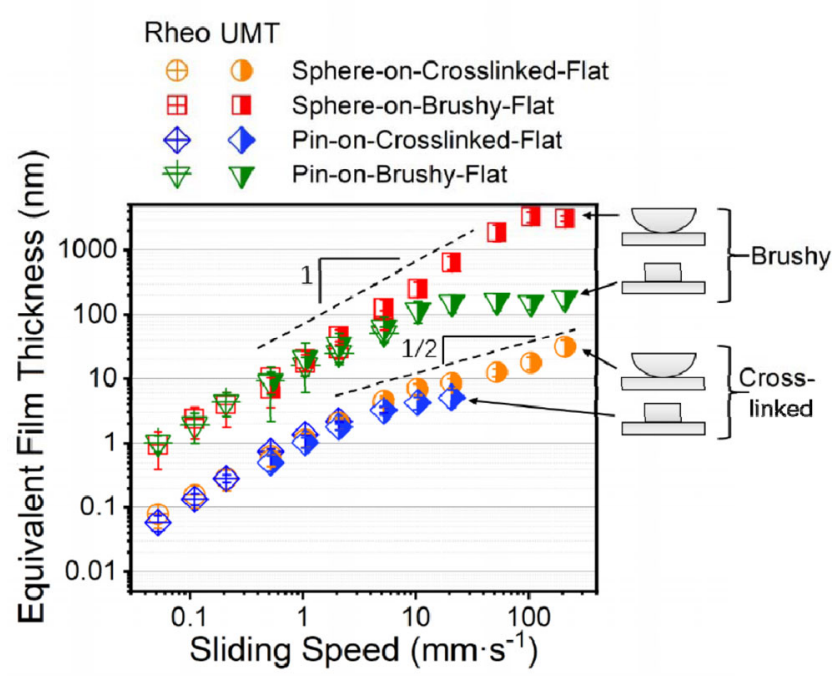

Fig. 5 Theoretical equivalent hydrodynamic film thickness as a function of speed for different contact geometries of PAAm hydrogels with different surfaces. The dashed lines show the scaling of the film thickness with sliding speed. It appears that a spherical contact enhances hydrodynamic film thickness compared to a flat contact, irrespective of the surface structure.

speeds above $10 \mathrm{~mm} \cdot \mathrm{s}^{-1}$, where the equivalent film thickness begins to level off. For the sphere on a crosslinked flat, owing to the enhancement of the water intake, the equivalent film thickness at sliding speeds above $10 \mathrm{~mm} \cdot \mathrm{s}^{-1}$ seems to increase with increasing sliding speeds to the power of 0.5 , which is similar to that predicted by (elasto) hydrodynamic theory $[36,38]$. For hydrogels with brushy surfaces, on the other hand, due to the sparse structure of the brushy gels, water is also flowing within the brushy layer. Thus, the equivalent hydrodynamic thickness includes both the gap between the outer extremities of the sliding hydrogels and the depth within which the water is flowing within the sparse, brushy-gel structure. The assumed surface roughness was around $5 \mathrm{~nm}$ [22], meaning that the $\lambda$ value would be larger than 1 at sliding speeds above $5 \mathrm{~mm} \cdot \mathrm{s}^{-1}$. Therefore, hydrodynamic effects are expected to play a dominant role in brushy-hydrogel friction at these high speeds. For hydrogels with the brushy surface in a flat-pinon-flat configuration, the equivalent film thickness appears to remain almost constant at high speeds, which could be due to the absence of a convergent contact inlet that would enable a wedge formation, as in the case of a sphere on flat. Therefore, the convergent inlet zone ahead of the sphere-on-flat contact apparently enhances the water supply, promotes rehydration in the near-surface region, and thus reduces the friction. Moreover, it is also important to mention that the viscosity of water in such thin layers might rise well above the nominal bulk viscosity of water due to the proximity of the polymer network, and is often referred to as bound-water viscosity [39]. According to Newton's law of viscous flow, a higher viscosity at given shear stress would mean a larger shearing thickness. This would, in turn, increase the $\lambda$ values, which would mean that the hydrodynamic forces could dominate friction at much lower sliding speeds. Moreover, considering the low elastic modulus of the PAAm hydrogels, the surface asperities may flatten significantly under the contact pressure, which would again increase the $\lambda$ parameter and support the formation of a thin, continuous lubrication layer between the sliding hydrogel surfaces at much lower sliding speeds. Therefore, hydrodynamic effects might be in play, even from the lowest tested sliding speeds.

To study the effect of contact pressure on COF and shear stress, experiments were conducted at different loads, and the results are displayed in Fig. 6. For the crosslinked hydrogels in both contact configurations, the trend of the COF-speed curve at $2 \mathrm{~N}$ was similar to that at $0.5 \mathrm{~N}$ (Fig. 6(a)). According to Hertzian contact theory, as the load is increased, the diameter of the contact area of the hydrogel sphere would increase from $8 \mathrm{~mm}$ to 12.5 $\mathrm{mm}$, and the contact pressure from 10 to $\sim 16 \mathrm{kPa}$. After normalizing the friction force by the contact area, as shown in Fig. 6(b), the shear stress of a sphere-on-crosslinked-flat configuration was higher at $2 \mathrm{~N}$ compared to $0.5 \mathrm{~N}$ over the entire range of sliding speeds. For the flat-pin-on-crosslinked-flat configuration, the contact area at $2 \mathrm{~N}$ was considered the same as that at $0.5 \mathrm{~N}$. Thus, the shear stress of the flat-pin-on-crosslinked-flat was found to increase with increasing load to a larger extent than for the sphere.

Similar results were also achieved with PAAm hydrogels with brushy surfaces. For the tests in either a sphere-on-flat configuration or a flat-pin-on-flat configuration, the trend of the shear-stress curve at $2 \mathrm{~N}$ was almost the same as that at $0.5 \mathrm{~N}$. Similarly to the crosslinked case, higher values of shear stress 

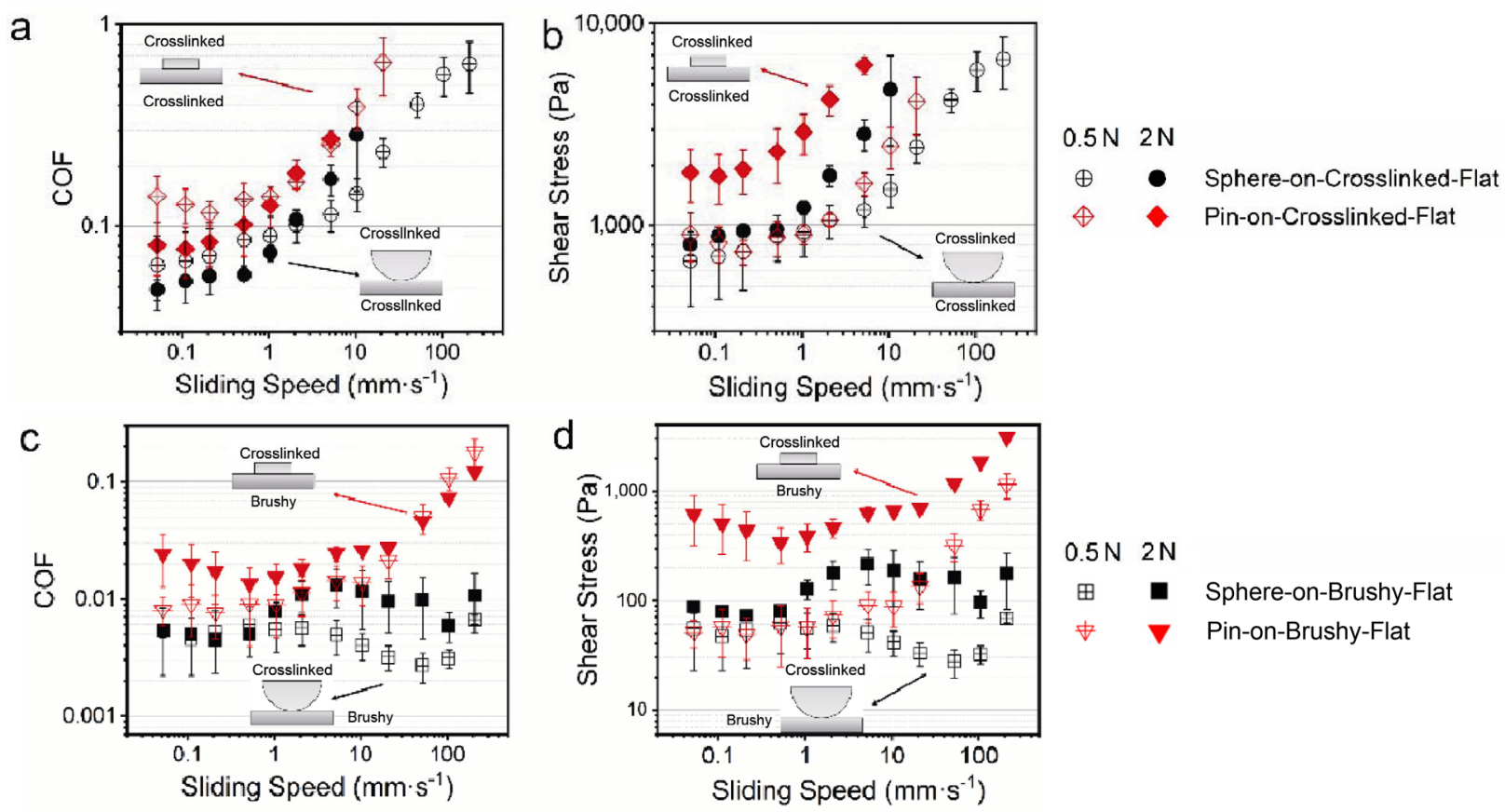

Fig. 6 Effect of load on (a) the COF and (b) the shear stress as a function of speed for the two contact geometries on a crosslinked hydrogel flat. (c, d) show the COF and the shear stress at different loads for both geometries on a brushy hydrogel flat. The tests performed at $0.5 \mathrm{~N}$ are shown by empty symbols and the tests performed at $2 \mathrm{~N}$ are shown by full symbols.

were measured for both contact geometries when the load was increased.

Since the shear stress was almost speed independent below $0.5 \mathrm{~mm} \cdot \mathrm{s}^{-1}$ for all cases, the average value of the shear stress at these speeds was calculated for each case and presented as a function of the corresponding average normal stress in Fig. 7. It was found that for hydrogels with crosslinked surfaces, the average low-speed shear stress was almost constant at normal stresses up to $16 \mathrm{kPa}$, at which point it started increasing rapidly with increasing normal stress. Similarly, the average low-speed shear stress of hydrogels with brushy surfaces only showed a slight increase at low normal stresses and increased more rapidly when the normal stress was increased to $20 \mathrm{kPa}$. The results thus indicate that the shear stress is not constant for different normal stresses. There seems to be a threshold normal stress, above which the shear stress starts increasing significantly. According to Simič et al. [23], the polymer concentration in the near-surface region of the brushy hydrogels increases with normal stress, while that of the crosslinked hydrogels is almost independent of normal stress below the osmotic pressure. Thus, it could be assumed that the shear stress of hydrogels at low speeds is probably dependent of the polymer density in the near-surface region, and the threshold pressure might be related to the osmotic pressure. Compared to the crosslinked gels, the shear stress of the brushy hydrogels increases more significantly with increasing normal stress due to the observed increase in polymer density in the surface region when normal stress is increased. At normal stresses above the osmotic pressure, the osmotic pressure cannot withstand the high normal stress, leading to serious densification of the near-surface region and a significant increase in the shear stress.

On the other hand, the shear stress at high speeds increases with increasing normal stress, which was most probably due to the thinner shearing thickness at higher loads, as also predicted by the elastohydrodynamic theory [38]. Schematic diagrams illustrating the differences in high-speed friction for a flat-pin-on- flat configuration and a sphere-on-flat configuration are shown in Fig. 8. However, since both geometries differ in their response at high speeds, the exact analysis of normal-stress dependence is not possible from data obtained at only two loads. 


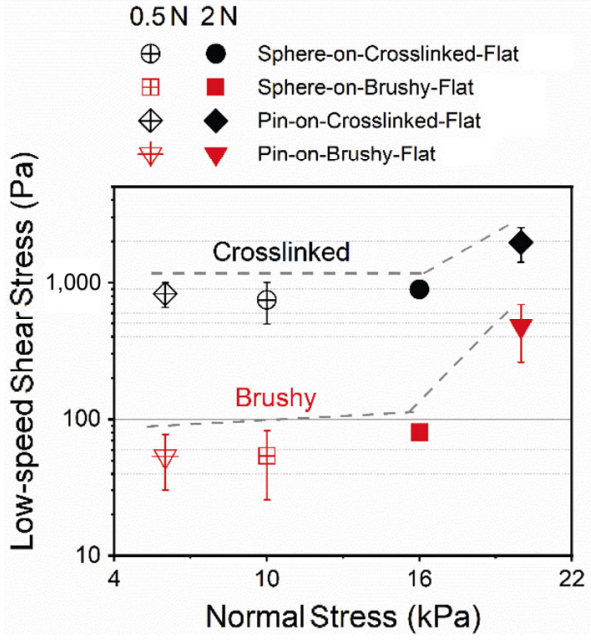

Fig. 7 Average shear stress at low speed as a function of the normal stress for different contact geometries of PAAm hydrogels with different surfaces. The dashed lines only show the variation trend of the shear stress with normal stress.

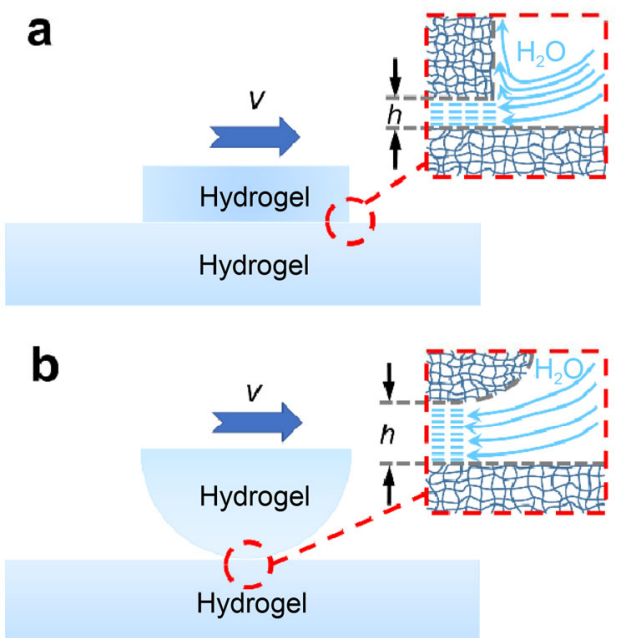

Fig. 8 Schematic diagrams of the probable cause for the differences in high-speed friction for (a) a flat-pin-on-flat configuration and (b) sphere-on-flat configuration. The convergent zone ahead of the sphere-on-flat contact enhances the water supply, promotes the speed-dependent film-thickness increase, and thus reduces the friction at high sliding speeds compared to the flat-pin-on-flat contact.

\section{Conclusions}

PAAm hydrogels with either crosslinked or brushy surfaces were synthesized by polymerization against the glass or PS molds, respectively, and tested in different contact geometries. Tribological experiments showed that brushy surfaces enabled up to an order-ofmagnitude lower friction compared to the crosslinked surfaces and even achieved superlubricity. After normalizing the friction force by the contact area, the shear stress of either crosslinked or brushy hydrogel surfaces at low sliding speeds appears to be equal for both contact geometries at a similar normal pressure. At high sliding speeds, which seem to correspond to $\lambda>1$, the shear stress and thus the COF was found to increase with sliding speed, which is characteristic for hydrodynamic effects. Moreover, at these high sliding speeds, a sphere-onflat configuration enabled lower shear stress compared to the flat-on-flat configuration. This is presumably due to the convergent contact inlet ahead of the sphere-on-flat contact, which enhances fluid-film formation and thus reduces hydrodynamic friction. The results in this work also show that shear stress of hydrogels at low sliding speeds is dependent on normal stress, which indicates that the polymer density in the near-surface region might play a role in lowspeed hydrogel friction. More direct polymer-chain contacts could occur in the contact region at higher loads, leading to an increase of the shear stress. Moreover, an increase in shear stress with increasing normal stress at higher sliding speeds was also observed, which could be due to the thinner shearing film at higher loads.

It seems that contact geometry and surface structure of hydrogels both play important roles in the tribological properties of hydrogels, and should be considered in future studies and applications.

\section{Acknowledgements}

This work was financially supported by the National Natural Science Foundation of China (Grant No. 51875303) and the European Research Council (ERC) under the European Union's Horizon 2020 research and innovation programme (Grant Agreement No 669562). Wenrui Liu wishes to acknowledge the Chinese Scholarship Council (CSC) for its support. The authors would also like to express their gratitude to Dr. André Brem for help with the rheometer setup.

Open Access This article is licensed under a Creative Commons Attribution 4.0 International License, which permits use, sharing, adaptation, distribution and 
reproduction in any medium or format, as long as you give appropriate credit to the original author(s) and the source, provide a link to the Creative Commons licence, and indicate if changes were made

The images or other third party material in this article are included in the article's Creative Commons licence, unless indicated otherwise in a credit line to the material. If material is not included in the article's Creative Commons licence and your intended use is not permitted by statutory regulation or exceeds the permitted use, you will need to obtain permission directly from the copyright holder.

To view a copy of this licence, visit http://creativecommons.org/licenses/by/4.0/.

\section{References}

[1] Gong J P. Friction and lubrication of hydrogels-Its richness and complexity. Soft Matter 2(7): 544-552 (2006)

[2] Ganji F, Vasheghani-Farahani S, Vasheghani-Farahani E. Theoretical description of hydrogel swelling: A review. Iran Polym J 19(5): 375-398 (2010)

[3] Mahinroosta M, Farsangi Z J, Allahverdi A, Shakoori Z. Hydrogels as intelligent materials: a brief review of synthesis, properties and applications. Mater Today Chem 8: 42-55 (2018)

[4] Lin C H, Hsu S H, Su J M, Chen C W. Surface modification of poly ( $\varepsilon$-caprolactone) porous scaffolds using gelatin hydrogel as the tracheal replacement. $J$ Tissue Eng Regen Med 5(2): 156-162 (2011)

[5] Fu R M, Tu L J, Zhou Y H, Fan L, Zhang F M, Wang Z G, Xing J, Chen D F, Deng C L, Tan G X, et al. A tough and self-powered hydrogel for artificial skin. Chem Mater 31(23): 9850-9860 (2019)

[6] Yu Y, Yuk H, Parada G A, Wu Y, Liu X Y, Nabzdyk C S, Youcef-Toumi K, Zang J F, Zhao X H. Multifunctional "hydrogel skins" on diverse polymers with arbitrary shapes. Adv Mater 31(7): 1807101 (2019)

[7] Kosukegawa H, Mamada K, Kuroki K, Liu L, Inoue K, Hayase T, Ohta M. Measurements of dynamic viscoelasticity of poly (vinyl alcohol) hydrogel for the development of blood vessel biomodeling. J Fluid Sci Technol 3(4): 533-543 (2008)

[8] Gong J P, Iwasaki Y, Osada Y, Kurihara K, Hamai Y. Friction of gels. 3. Friction on solid surfaces. J Phys Chem B 103(29): 6001-6006 (1999)

[9] Gong J P, Kagata G, Iwasaki Y, Osada Y. Surface friction of polymer gels: 1. Effect of interfacial interaction. Wear 251(1-12): 1183-1187 (2001)

[10] Gong J P, Kurokawa T, Narita T, Kagata G, Osada Y,
Nishimura G, Kinjo M. Synthesis of hydrogels with extremely low surface friction. J Am Chem Soc 123(23): 5582-5583 (2001)

[11] Kagata G, Gong J P, Osada Y. Friction of gels. 6. Effects of sliding velocity and viscoelastic responses of the network. J Phys Chem B 106(18): 4596-4601 (2002)

[12] Gong J P, Osada Y. Gel friction: a model based on surface repulsion and adsorption. J Chem Phys 109(18): 8062-8068 (1998)

[13] Nonoyama T, Gong J P. Double-network hydrogel and its potential biomedical application: a review. Proc Inst Mech Eng H 229(12): 853-863 (2015)

[14] Ohsedo Y, Takashina R, Gong J P, Osada Y. Surface friction of hydrogels with well-defined polyelectrolyte brushes. Langmuir 20(16): 6549-6555 (2004)

[15] Kii A, Xu J, Gong J P, Osada Y, Zhang X M. Heterogeneous polymerization of hydrogels on hydrophobic substrate. J Phys Chem B 105(20): 4565-4571 (2001)

[16] Rennie A C, Dickrell P L, Sawyer W G. Friction coefficient of soft contact lenses: Measurements and modeling. Tribol Lett 18(4): 499-504 (2005)

[17] Urueña J M, McGhee E O, Angelini T E, Dowson D, Sawyer W G, Pitenis A A. Normal load scaling of friction in gemini hydrogels. Biotribology 13: 30-35 (2018)

[18] Pitenis A A, Urueña J M, Schulze K D, Nixon R M, Dunn A C, Krick B A, Sawyer W G, Angelini T E. Polymer fluctuation lubrication in hydrogel gemini interfaces. Soft Matter 10(44): 8955-8962 (2014)

[19] Urueña J M, Pitenis A A, Nixon R M, Schulze K D, Angelini T E, Sawyer W G. Mesh size control of polymer fluctuation lubrication in gemini hydrogels. Biotribology 1-2: 24-29 (2015)

[20] Reale E R, Dunn A C. Poroelasticity-driven lubrication in hydrogel interfaces. Soft Matter 13(2): 428-435 (2017)

[21] Pitenis A A, Sawyer W G. Lubricity of high water content aqueous gels. Tribol Lett 66(3): 113 (2018)

[22] Meier Y A, Zhang K H, Spencer N D, Simič R. Linking friction and surface properties of hydrogels molded against materials of different surface energies. Langmuir 35(48): 15805-15812 (2019)

[23] Simič R, Yetkin M, Zhang K H, Spencer N D. Importance of hydration and surface structure for friction of acrylamide hydrogels. Tribol Lett 68(2): 64 (2020)

[24] Klein J. Hydration lubrication. Friction 1(1): 1-23 (2013)

[25] Jiang C K, Jiang H, Zhang J W, Kang G Z. Analytical model of friction behavior during polymer scratching with conical tip. Friction 7(5): 466-478 (2019)

[26] Popov V L, Pohrt R, Li Q. Strength of adhesive contacts: influence of contact geometry and material gradients. Friction 5(3): 308-325 (2017)

[27] Moore A C, Burris D L. Tribological rehydration of cartilage and its potential role in preserving joint health. 
Osteoarthr Cartil 25(1): 99-107 (2017)

[28] Zhang K H, Simič R, Yan W Q, Spencer N D. Creating an interface: rendering a double-network hydrogel lubricious via spontaneous delamination. ACS Appl Mater Interfaces 11(28): 25427-25435 (2019)

[29] Fairbanks B D, Schwartz M P, Bowman C N, Anseth K S. Photoinitiated polymerization of PEG-diacrylate with lithium phenyl-2,4,6-trimethylbenzoylphosphinate: polymerization rate and cytocompatibility. Biomaterials 30(35): 6702-6707 (2009)

[30] Majima T, Schnabel W, Weber W. Phenyl-2,4,6-trimethylbenzoylphosphinates as water-soluble photoinitiators. Generation and reactivity of $\mathrm{O}=\dot{\mathrm{P}}\left(\mathrm{C}_{6} \mathrm{H}_{5}\right)\left(\mathrm{O}^{-}\right)$radical anions. Die Makromol Chem 192(10): 2307-2315 (1991)

[31] Sader J E, Chon J W M, Mulvaney P. Calibration of rectangular atomic force microscope cantilevers. Rev Sci Instrum 70(10): 3967-3969 (1999)

[32] Cannara R J, Eglin M, Carpick R W. Lateral force calibration in atomic force microscopy: A new lateral force calibration method and general guidelines for optimization. Rev Sci Instrum 77(5): 053701 (2006)

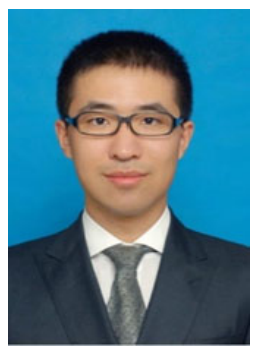

Wenrui LIU. He received his bachelor degree from the University of Science and Technology Beijing, China, in 2016. He is currently a Ph.D. student in the State Key Laboratory of Tribology at Tsinghua

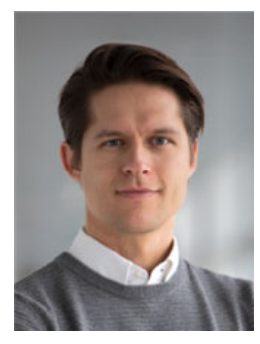

Rok SIMIČ. He obtained his bachelor degree in physics in 2009 and a Ph.D. degree in mechanical engineering in 2013, both at the University of Ljubljana, Slovenia. During his Ph.D. period, he worked in the group of Prof. Mitjan Kalin studying lubrication of diamond-like carbon coatings. Since 2015, he has been a postdoctoral researcher in
[33] Hertz H. On the contact of elastic solids. J für die Reine und Angew Math 92: 156-171 (1881)

[34] Johnson K L. Contact Mechanics. Cambridge (UK): Cambridge University Press, 1985.

[35] Delavoipière J, Tran Y, Verneuil E, Heurtefeu B, Hui C Y, Chateauminois A. Friction of poroelastic contacts with thin hydrogel films. Langmuir 34(33): 9617-9626 (2018)

[36] Martin A, Clain J, Buguin A, Brochard-Wyart F. Wetting transitions at soft, sliding interfaces. Phys Rev E 65(3): 031605 (2002)

[37] Kurokawa T, Tominaga T, Katsuyama Y, Kuwabara R, Furukawa H, Osada Y, Gong J P. Elastic-hydrodynamic transition of gel friction. Langmuir 21(19): 8643-8648 (2005)

[38] Hamrock B J, Dowson D. Elastohydrodynamic lubrication of elliptical contacts for materials of low elastic modulus I-Fully flooded conjunction. J Lubr Technol 100(2): 236-245 (1978)

[39] Raviv U, Laurat P, Klein J. Fluidity of water confined to subnanometre films. Nature 413(6851): 51-54 (2001)

University, China. From August 2019 to June 2020, he worked with Prof. Nicholas D. Spencer as a visiting Ph.D. student at the Laboratory of Surface Science and Technology at ETH Zurich, Switzerland. His research interests include hydrogel friction, liquid superlubricity, and surface $\&$ interface analysis.

the group of Prof. Nicholas D. Spencer at ETH Zurich. The focus of his current work lies on soft matter, in particular friction, surfaces, and mechanics of hydrogels. His research spans from the fundamental science of hydrogels to biomedical applications such as contact lenses. He has published 20 peer-reviewed papers, supervised several postgraduate and Ph.D. students, and held training courses and coordinated projects. 


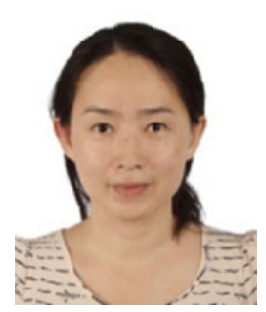

Yuhong LIU. She received her Ph.D. degree in CAS Key Laboratory of Molecular Nanostructure \& Nanotechnology Institute of Chemistry, Chinese Academy of Sciences, Beijing, China, in 2005. She joined the State Key

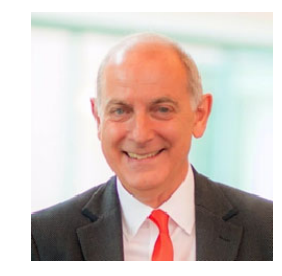

Nicholas D. SPENCER. He is a professor of surface science and technology, ETH Zurich, obtained his bachelor and Ph.D. degrees at the University of Cambridge, before working as a postdoc at the University of California, Berkeley. After a decade in the US chemical industry, he joined the ETH Zurich in 1993, where he has served as both the head of the Department of Materials and the President of the Research Commission. He is co-founder of Swiss
Laboratory of Tribology at Tsinghua University in 2005. Her current position is an associate professor. Her research areas cover nanotribology, nanostructure \& nanotechnology of surface and interface, chemical-mechanical planarization, and water-based lubrication.

Tribology and the founder and Chair of the International Tribology Forum. He is also co-founding Editor and Editor in Chief of the journal Tribology Letters. He is a member of the Swiss Academy of Engineering Sciences, a fellow of both the Royal Society of Chemistry (UK) and the Society for Tribologists and Lubrication Engineers, and was awarded the 2018 Tribology Gold Medal. He has published over 440 papers in academic journals, edited several monographs, and holds over a dozen patents. 